\title{
Safety and efficacy of nurse-controlled analgesia in patients less than I year of age
}

\author{
Hina Walia' \\ Dmitry Tumin' \\ Sharon Wrona' \\ David Martin ${ }^{1,2}$ \\ Tarun Bhalla ${ }^{1,2}$ \\ Joseph D Tobias ${ }^{1-3}$ \\ 'Department of Anesthesiology \\ and Pain Medicine, Nationwide \\ Children's Hospital, ${ }^{2}$ Department of \\ Anesthesiology and Pain Medicine, \\ ${ }^{3}$ Department of Pediatrics, The Ohio \\ State University College of Medicine, \\ Columbus, $\mathrm{OH}$, USA
}

Correspondence: Hina Walia

Department of Anesthesiology and Pain Medicine, Nationwide Children's Hospital, 700 Children's Drive,

Columbus, OH 43205, USA

$\mathrm{Tel}+\mathrm{I} 6147224200$

Fax +I 6I47224203

Email hina.walia@nationwidechildrens.org

\author{
This article was published in the following Dove Press journal: \\ Journal of Pain Research \\ II June 2016 \\ Number of times this article has been viewed
}

Background: The management of acute pain presents unique challenges in the younger pediatric population. Although patient-controlled devices are frequently used in patients $\geq 6$ years of age, alternative modes of analgesic delivery are needed in infants.

Objective: To examine the safety and efficacy of nurse-controlled analgesia (NCA) in neonates less than 1 year of age.

Methods: Data from patients $<1$ year of age receiving NCA as ordered by the Acute Pain Service at our institution were collected over a 5-year period and reviewed retrospectively. The primary outcomes were activation of the institution's Rapid Response Team (RRT) or Code Blue, signifying severe adverse events. Pain score after NCA initiation was a secondary outcome.

Results: Among 338 girls and 431 boys, the most common opioid used for NCA was fentanyl, followed by morphine and hydromorphone. There were 39 (5\%) cases involving RRT or Code Blue activation, of which only one (Code Blue) was activated due to a complication of NCA (apnea). Multivariable logistic regression demonstrated morphine NCA to be associated with greater odds of RRT activation $(\mathrm{OR}=3.29,95 \% \mathrm{CI}=1.35,8.03, P=0.009)$ compared to fentanyl NCA. There were no statistically significant differences in pain scores after NCA initiation across NCA agents.

Conclusion: NCA is safe in neonates and infants, with comparable efficacy demonstrated for the three agents used. The elevated incidence of RRT activation in patients receiving morphine suggests caution in its use and consideration of alternative agents in this population.

Keywords: nurse-controlled analgesia, pain medicine, Rapid Response Team

\section{Introduction}

The pediatric population is at risk of poor pain assessment and management which in turn may result in immediate and long-term adverse physiologic effects. ${ }^{1-5}$ The continuous infusion of opioids remains the mainstay of analgesic therapy in neonates and infants. ${ }^{6-8}$ The safety of opioids for critically ill and/or preterm neonates and infants has not been well defined..$^{7-9}$ Furthermore, there are limited data demonstrating the optimal opioid or mode of delivery in this population. Opioid dosing in these populations is complicated by differences in body composition, drug absorption, distribution, metabolism and elimination, variability in the severity of illness, combined with rapid changes in brain development, receptor expression, opioid pharmacodynamics, and pain mechanisms. ${ }^{10,11}$

Although patient-controlled devices are frequently used in patients who are 6 years of age and older, alternative modes of delivery are needed in infants and young children. Nurse-controlled analgesia (NCA) has the potential to improve pain management by 
allowing more involvement of caregivers in the treatment process and allowing the immediate treatment of pain based on the health care provider's assessment. NCA allows for flexible dosing, rapid titration in response to pain or the anticipation of pain, increased health care provider (nursing) participation, and decreased need to repeatedly breach the intravenous site. ${ }^{12}$ Although the safety and efficacy of NCA have been demonstrated in several pediatric populations, there are limited data in neonates and infants. ${ }^{12-14}$ The present study retrospectively reviews patients less than 1 year of age who received NCA as ordered by the Acute Pain Service (APS) at our institution over a 5-year period. The focal outcomes included adverse effects necessitating activation of a Rapid Response Team (RRT) or Code Blue in this patient population. We examine the incidence of these adverse events among neonates and infants receiving NCA, and test for potential associations of adverse events with NCA agents and patient characteristics.

\section{Methods}

This retrospective study was approved by the Nationwide Children's Hospital Institutional Review Board with the waiver for the need to obtain written and informed consent being due to minimal risk to the subjects and as the research could not have reasonably been carried out without the waiver. This study involved a retrospective review of the quality improvement database from the Department of Anesthesiology and Pain Medicine at Nationwide Children's Hospital (Columbus, Ohio). Nationwide Children's Hospital is an urban, free-standing, 510-bed tertiary care children's hospital. The database consisted of electronic medical records of patients with anesthetic procedures between January 2010 and October 2015. Each patient less than 1 year of age receiving NCA was identified and the hospital records were reviewed. Data on patient characteristics including age, weight, sex, events of interest (RRT or Code Blue), and NCA agents used were obtained for analysis. At our institution, the RRT is activated at any time when the physician team, nurse, or parent are concerned about the patient's status or safety, whereas Code Blue is activated when there is an acute change in cardiorespiratory status or a cardiopulmonary arrest. The analysis of RRT activation captures potential complications of NCA, such as excessive dosing resulting in respiratory depression, nausea, or vomiting. Data on pain scores after NCA initiation were available for infants $<1$ year of age receiving NCA since September 2014 due to restrictions by electronic medical record implementation.

For each patient, data from the earliest admission involving an encounter with the APS were analyzed. Each admission may have included a single encounter with the APS at which NCA was initiated, or may have included multiple encounters at which NCA was monitored, and in some cases, changed to another agent (fentanyl, morphine, hydromorphone). The admissions were classified as involving a single encounter with the APS; involving multiple encounters with no change in NCA agent; or involving multiple encounters with a change from the initial NCA agent used. Events of interest (ie, RRT or Code Blue activation) were tracked from the time of the index admission, with patients labeled as experiencing no event, RRT activation (including a subsequent Code Blue, if applicable), or Code Blue activation alone. If available, the first pain score after the initiation of NCA obtained using the FLACC (face, legs, activity, cry, consolability) scale was selected for analysis.

Patient demographic characteristics, changes in NCA medication, RRT or Code Blue events, and pain scores were compared by the type of first NCA agent using chi-square tests for categorical variables and analysis of variance for continuous variables. Multivariable logistic regression was used to examine the influence of NCA agent on odds of an RRT or Code Blue event adjusting for potential confounding variables, including patient age, sex, and weight. Statistical analysis was performed using Stata/IC 13.0 (StataCorp LP, College Station, TX, USA) and $P<0.05$ was considered statistically significant for all analyses.

\section{Results}

The study cohort included 769 infants who received an APS consult between January 2010 and October 2015. Of these 769 patients, 766 received fentanyl, morphine, or hydromorphone as the first NCA agent, while three patients had an unknown first NCA agent. The patients ranged in age from 0 to 362 days (145.0 \pm 101.0 days) and in weight from 1.9 to $14.0 \mathrm{~kg}(5.7 \pm 2.0 \mathrm{~kg})$. There were $338(44 \%)$ girls and 431 $(56 \%)$ boys. The most common opioid used for NCA was fentanyl (69\%), followed by morphine (20\%) and hydromorphone (11\%) (Table 1). Statistically significant differences in patient demographics by NCA type included patients receiving fentanyl having the youngest age and lowest weight when comparing the three opioids. Sex composition did not differ by NCA type. There were more patients whose NCA agent was changed in the morphine group (15 of 151, $10 \%$ ) compared to the hydromorphone (3 of $84,4 \%)$ and fentanyl (13 of 531, 2\%) groups $(P<0.001)$. Patients with multiple encounters, but no change in NCA agent included 110 of $151(73 \%)$ in the morphine group, 60 of $84(71 \%)$ in the hydromorphone group, and 408 of 531 (77\%) in the fentanyl group. 
Table I Characteristics of infants receiving nurse-controlled analgesia, by first analgesic agent $(\mathrm{N}=766)$

\begin{tabular}{|c|c|c|c|c|}
\hline Variables & Fentanyl ( $N=53 I)$ & Hydromorphone $(\mathbf{N}=84)$ & Morphine $(\mathrm{N}=|5|)$ & $P$-value \\
\hline Male, n (\%) & $297(56)$ & $46(55)$ & $88(58)$ & $\mathrm{n} / \mathrm{s}$ \\
\hline Age (days), mean (SD) & $122.0(98.0)$ & $204.6(75.0)$ & $195.0(92.7)$ & $* * *$ \\
\hline Weight $(\mathrm{kg})$, mean (SD) & $5.2(1.9)$ & $6.9(1.6)$ & $6.9(1.9)$ & $* * *$ \\
\hline \multicolumn{5}{|l|}{ Pain service encounters } \\
\hline One, n (\%) & $110(21)$ & $21(25)$ & $26(17)$ & $\mathrm{n} / \mathrm{s}$ \\
\hline Multiple, no change in NCA, $n(\%)$ & $408(77)$ & $60(7 I)$ & $110(73)$ & $\mathrm{n} / \mathrm{s}$ \\
\hline Multiple, NCA changed, n (\%) & $13(2)$ & $3(4)$ & $15(10)$ & $* * *$ \\
\hline \multicolumn{5}{|l|}{ Adverse events } \\
\hline RRT activation, n (\%) & $12(2)$ & $2(2)$ & $12(8)$ & $* *$ \\
\hline Code Blue, n (\%) & $10(2)$ & $I(I)$ & $2(1)$ & $n / s$ \\
\hline
\end{tabular}

Note: $P$-values by chi-square test for categorical variables and ANOVA for continuous variables: $n / s, P>0.05 ; * * P<0.01 ; * * * P<0.001$.

Abbreviations: SD, standard deviation; NCA, nurse-controlled analgesia; n/s, not significant; RRT, Rapid Response Team; ANOVA, analysis of variance.

The RRT was activated for 26 patients (3\%) and a Code Blue was called for 13 patients (2\%). Four cases where an RRT was called prior to Code Blue were classified in the RRT group. RRT events were significantly more common for morphine NCA (12 of 151, 8\%) compared to fentanyl NCA (12 of 531, 2\%) or hydromorphone NCA ( 2 of $84,2 \%$; $P=0.003)$. There were no differences in Code Blue events by NCA type ( $P=0.833)$. Major reasons for activating either type of response team included respiratory concerns (17 of 26 RRT and 5 of 13 Code Blue) and cardiovascular concerns (5 of 26 RRT and 2 of 13 Code Blue). The most common respiratory concerns included tachypnea or trouble breathing. The most common cardiovascular concerns include arrhythmia and increased heart rate. Events originating primarily due to complications of NCA were rare, with only one Code Blue and no RRTs being initiated due to what was subsequently determined to be a complication of NCA (Table 2). Following five RRTs and no Code Blue events, the type of NCA was changed. These included one change from fentanyl to hydromorphone and four changes from morphine to another opioid. Morphine was changed to fentanyl in two cases and to hydromorphone in two others. No statistically significant differences in the reason for an event were identified when comparing RRT and Code Blue ( $P=0.159)$ events.

The analytic sample for pain score data included 198 patients receiving NCA since September 2014, of whom 156 had data on the first pain score within 24 hours of NCA initiation. In this group, $71 \%(110 / 156)$ received fentanyl, $24 \%$ (37/156) received morphine, and 5\% (8/156) received hydromorphone. No difference in the FLACC-based pain score after NCA initiation was noted when comparing the three opioids with pain scores of $1.2 \pm 2.1,1.4 \pm 2.5$, and $1.1 \pm 2.1$, respectively, in patients receiving fentanyl, morphine, and hydromorphone. An independent $t$-test demonstrated no
Table 2 Reasons for RRT or Code Blue activation ( $N=39)$

\begin{tabular}{lll}
\hline Reason & RRT & Code Blue \\
\hline Cardiovascular & $19 \%$ & $8 \%$ \\
& 2 Hydromorphone & I Fentanyl \\
& 3 Morphine & I Hydromorphone \\
Respiratory & $65 \%$ & $38 \%$ \\
& I Fentanyl & 5 Fentanyl \\
& 7 Hydromorphone & \\
NCA related & 9 Morphine & $4 \%$ \\
& 0 & I Morphine \\
Other & & $38 \%$ \\
& $15 \%$ & 4 Fentanyl \\
& I Hydromorphone & I Morphine
\end{tabular}

Note: Data shown as number or \%.

Abbreviations: RRT, Rapid Response Team; NCA, nurse-controlled analgesia.

statistically significant difference in average first pain scores between patients who had an RRT or Code Blue activation (1.2 \pm 2.3$)$ and patients who did not (1.3 $\pm 2.2 ; P=0.854)$.

Multivariable logistic regression models for outcomes of RRT (Model 1) and Code Blue (Model 2) activation after adjusting for sex, age, and weight are presented in Table 3. In each model, the reference group includes all patients not experiencing the outcome event. Model 1 confirmed increased odds of RRT activation with morphine as opposed to fentanyl $(\mathrm{OR}=3.29,95 \% \mathrm{CI}=1.35,8.03, P=0.009)$, with no statistically significant differences in odds of RRT activation between morphine and hydromorphone $(P=0.107)$, or hydromorphone and fentanyl $(P=0.939)$. Model 2 demonstrated no statistically significant influence of NCA type on the odds of a Code Blue being called. There were no statistically significant associations between either type of events and any of the demographic variables included in these models, demonstrating that demographic characteristics did not contribute to adverse effects in this cohort. Furthermore, a 
sub-analysis comparing RRT activation to Code Blue among patients experiencing either events $(n=39)$ identified no statistically significant differences by NCA type or by other characteristics in the odds of RRT rather than Code Blue being called (Table 4).

\section{Discussion}

Given the potential deleterious physiologic effects of untreated acute pain in neonates and infants, effective management strategies are needed. However, this population also experiences the highest risk of adverse effects. ${ }^{15}$ Patient controlled analgesia or NCA provides effective analgesia while limiting the total dose and adverse effects of opioids in toddlers and older children. Yet, our understanding of NCA safety in neonates and infants is limited by the number of studies examin-

Table 3 Multivariable logistic regression of adverse events among infants receiving NCA $(\mathrm{N}=764)$

\begin{tabular}{lllll}
\hline Variables & \multicolumn{2}{l}{ Model I: RRT } & \multicolumn{2}{l}{ Model 2: Code Blue } \\
\cline { 2 - 5 } & OR & $\mathbf{9 5 \%} \mathbf{~ C l}$ & OR & $\mathbf{9 5 \% ~ C I}$ \\
\hline First NCA agent & & & & \\
$\quad$ Fentanyl & Reference & & Reference & \\
Hydromorphone & 0.94 & $0.20,4.46$ & 0.97 & $0.11,8.46$ \\
$\quad$ Morphine & $3.29 * *$ & $1.35,8.03$ & 1.06 & $0.21,5.34$ \\
Male & 0.67 & $0.30,1.52$ & 1.33 & $0.42,4.23$ \\
Age (days) & 1.00 & $0.99,1.00$ & 1.00 & $0.99,1.01$ \\
Weight (kg) & 1.22 & $0.88,1.68$ & 0.70 & $0.40,1.25$ \\
\hline
\end{tabular}

Note: $* * P<0.01$.

Abbreviations: NCA, nurse-controlled analgesia; RRT, Rapid Response Team; $\mathrm{OR}$, odds ratio; $\mathrm{Cl}$, confidence interval.

Table 4 Multivariable logistic regression of RRT activation during $\mathrm{NCA}(\mathrm{N}=39)$

\begin{tabular}{lll}
\hline Variables & OR* & $\mathbf{9 5 \%} \mathbf{~ C l}$ \\
\hline First NCA agent & & \\
$\quad$ Fentanyl & Reference & \\
Hydromorphone & 0.82 & $0.05,14.14$ \\
$\quad$ Morphine & 4.17 & $0.57,30.64$ \\
Male & 0.26 & $0.04,1.55$ \\
Age (days) & 1.00 & $0.98,1.01$ \\
Weight (kg) & 1.82 & $0.83,3.95$ \\
\hline
\end{tabular}

Note: *Odds ratio of RRT rather than Code Blue being called for infants experiencing an adverse event.

Abbreviations: RRT, Rapid Response Team; NCA, nurse-controlled analgesia; $\mathrm{OR}$, odds ratio; $\mathrm{Cl}$, confidence interval. ing NCA in this population. Our current study is the first to assess the use of NCA in a pediatric population less than 1 year of age. We examined three different opioids for NCA, which were administered with continuous doses and variable lockout intervals. Our retrospective review over a 5-year period demonstrated the safety of NCA in a large cohort of patients with a very limited adverse effect profile. While a retrospective review might theoretically miss low-risk adverse events such as nausea or pruritus, our approach of tracking the activation of the RRT or Code Blue responses clearly demonstrates the low risk of NCA in this population. RRT $(n=26)$ and Code Blue ( $n=13)$ events were rare ( $2 \%$ and $3 \%$, respectively) among patients younger than 1 year who were receiving NCA, and there was only one Code Blue activation that was definitively related to a complication of NCA. However, there were more RRT activations when using morphine as the primary opioid as compared to fentanyl and hydromorphone.

Unlike previous studies, given the younger age of the current cohort, fentanyl was the primary agent used in these patients. Although morphine or hydromorphone have been the opioids used most commonly for patient controlled analgesia or NCA, there is growing clinical experience with the use of fentanyl NCA, especially in neonates or young infants, given its more stable pharmacokinetic profile and perhaps lower risk of adverse effects including respiratory depression. ${ }^{16}$ Our institution's current guidelines for dosing for fentanyl, hydromorphone, and morphine NCA are described in Table 5. Our experience, institutional culture, and preference for a short-acting agent with more predictable pharmacokinetics, have led us to prefer fentanyl for NCA in neonates and infants. This is reflected in the findings in the current study with approximately $70 \%$ of the patients receiving fentanyl as the primary agent for NCA.

Furthermore, the current review provides data suggesting that fentanyl may offer specific advantages over morphine as a greater percentage of patients who started with morphine NCA required a switch to a second agent. In a retrospective review of 514 cases involving the entire spectrum of the pediatric age range, we noted that more patients who were initially started on morphine were switched to either hydromorphone or fentanyl. ${ }^{17}$ Of the 17 morphine-to-hydromorphone switches with adverse effects,

Table 5 NCA dosing guidelines at Nationwide Children's Hospital

\begin{tabular}{llll}
\hline NCA guidelines & Morphine & Hydromorphone & Fentanyl \\
\hline Initial bolus dose & $0.03-0.05 \mathrm{mg} / \mathrm{kg} /$ dose & $0.01-0.015 \mathrm{mg} / \mathrm{kg} /$ dose & $0.5-1 \mu \mathrm{gg} / \mathrm{kg}$ \\
NCA dose & $0.2 \mathrm{mg} / \mathrm{kg}$ & $0.004 \mathrm{mg} / \mathrm{kg}$ & $0.5 \mu \mathrm{g} / \mathrm{kg}$ \\
Lockout interval & $10-30$ minutes & $10-30 \mathrm{minutes}$ & $10-20 \mathrm{minutes}$ \\
Continuous dose & $0.02 \mathrm{mg} / \mathrm{kg} / \mathrm{h}$ & $0.004 \mathrm{mg} / \mathrm{kg} / \mathrm{h}$ & $0.5 \mu \mathrm{g} / \mathrm{kg}$ \\
\hline
\end{tabular}

Abbreviation: NCA, nurse-controlled analgesia. 
pruritus (64.7\%) and inadequate pain control (47.1\%) were the most common side effects. The most common side effect resulting in a hydromorphone-to-morphine switch was nausea $(66.7 \%)$. However, when pain scores were available, the current data demonstrate no difference in the pain scores during the initial 24 hours of NCA when comparing fentanyl, morphine, and hydromorphone.

The major advantage of the current study is that it used institutionally defined criteria for severe adverse events, as indicated by the activation of the Code Blue and RRT. One limitation of this approach is that we may have missed complications of NCA not severe enough to lead to activation of these teams. Another potential limitation of the database used may be inconsistent attribution of adverse events to complications of NCA. However, this was supplemented by noting when a change in the opioid prescription was required. For example, five RRT events occurred in patients where the NCA agent was changed (four of five of these events resulted in a switch from morphine to another opioid). However, in review, none of the RRTs were primarily attributed to NCArelated causes. The limitations of our data precluded more detailed investigation of the safety and side effects of changes in NCA agents. While non-life-threatening adverse effects may be lost in retrospective reviews of the hospital records, bothersome adverse effects or inadequate analgesia would be identified accurately as a medication change.

\section{Conclusion}

Authorized Agent-Controlled Analgesia (AACA) offers many advantages to pain management in children. At our institution, NCA is the standard form of AACA used in patients $<1$ year of age. This retrospective review demonstrates the general safety of NCA in infants by investigating events of response team activation (RRT or Code Blue) among patients receiving NCA. In our cohort of more than 700 patients, only one such activation was determined to be directly related to NCA. The rarity of these adverse events demonstrates the safety of NCA in this age range. Additionally, we noted that the need to switch to a different opioid was higher in patients started on morphine NCA when compared to fentanyl NCA, thereby suggesting that fentanyl may offer a more acceptable adverse effect profile. In our current practice, fentanyl (dosed as in Table 5) is the most frequently used agent for NCA because of its short duration of action and favorable adverse effect profile. We found no difference in pain scores when comparing the three commonly used opioids for NCA (fentanyl, morphine, and hydromorphone). Future prospective studies evaluating the efficacy and safety of the various opioids and dosing regimens for NCA will add to our understanding of pain management in the neonatal and infant population.

\section{Disclosure}

The authors report no conflicts of interest in this work.

\section{References}

1. Ellis JA, O'Connor V, Cappelli M, et al. Pain in hospitalized pediatric patients: how are we doing? Clin J Pain. 2002;18(4):262-269.

2. Weisman SJ, Bernstein B, Schecter NL. Consequences of inadequate analgesia during painful procedures in children. Arch Pediatr Adolesc Med. 1998;152(2):147-149.

3. Johnston CC, Stevens BJ. Experience in a neonatal intensive care unit affects pain response. Pediatrics. 1996;98(5):925-930.

4. Craig KD, Lilley CM, Gilbert CA. Barriers to optimal pain management in infants, children, and adolescents social barriers to optimal pain management in infants and children. Clin J Pain. 12(3):232-242.

5. Franck LS, Cox S, Allen A, et al. Parental concern and distress about infant pain. Arch Dis Child Fetal Neonatal Ed. 2004;89(1):F71-F75.

6. De Lima J, Carmo KB. Practical pain management in the neonate. Best Pract Res Clin Anaesthesiol. 2010;24(3):291-307.

7. Van Dijk M, Bouwmeester NJ, Duivenvoorden HJ, et al. Efficacy of continuous versus intermittent morphine administration after major surgery in 0-3-year-old infants; a double-blind randomized controlled trial. Pain. 2002;98(3):305-313.

8. American Academy of Pediatrics. Prevention and management of pain and stress in the neonate. American Academy of Pediatrics. Committee on Fetus and Newborn. Committee on Drugs. Section on Anesthesiology. Section on Surgery. Canadian Paediatric Society. Fetus and Newborn Committee. Pediatrics. 2000;105(2):454-461.

9. Anand KJ, Hall RW. Pharmacological therapy for analgesia and sedation in the newborn. Arch Dis Child Fetal Neonatal Ed. 2006;91(6):F448-F453.

10. Taddio A, Katz J. Pain, opioid tolerance and sensitisation to nociception in the neonate. Best Pract Res Clin Anaesthesiol. 2004;18(2): 291-302.

11. Simons SH, Anand KJ. Pain control: opioid dosing, population kinetics and side-effects. Semin Fetal Neonatal Med. 2006;11(4):260-267.

12. Czarnecki ML, Ferrise AS, Jastrowski Mano KE, et al. Parent/nursecontrolled analgesia for children with developmental delay. Clin J Pain. 2008;24(9):817-824.

13. Anghelescu DL, Burgoyne LL, Oakes LL, et al. The safety of patientcontrolled analgesia by proxy in pediatric oncology patients. Anesth Analg. 2005;101(6):1623-1627.

14. Malviya S, Voepel-Lewis T, Tait AR, et al. Pain management in children with and without cognitive impairment following spine fusion surgery. Paediatr Anaesth. 2001;11(4):453-458.

15. Anand KJ, Aranda JV, Berde CB, et al. Summary proceedings from the neonatal pain-control group. Pediatrics. 2006;117(3 Pt 2):S9-S22.

16. Naguib AN, Dewhirst E, Winch PD, Simsic J, Galantowicz M, Tobias JD. Pain management after comprehensive stage II repair for hypoplastic left heart syndrome. Pediatr Cardiol. 2013;34(1):52-58.

17. DiGiusto M, Bhalla T, Martin D, Foerschler D, Jones MJ, Tobias JD. Patient-controlled analgesia in the pediatric population: morphine versus hydromorphone. J Pain Res. 2014;7:471-475. 


\section{Publish your work in this journal}

The Journal of Pain Research is an international, peer reviewed, open access, online journal that welcomes laboratory and clinical findings in the fields of pain research and the prevention and management of pain. Original research, reviews, symposium reports, hypothesis formation and commentaries are all considered for publication.

Submit your manuscript here: https://www.dovepress.com/journal-of-pain-research-journal

The manuscript management system is completely online and includes a very quick and fair peer-review system, which is all easy to use. Visit http://www.dovepress.com/testimonials.php to read real quotes from published authors. 